\title{
TENDÊNCIAS DA PRODUÇÃO DO CONHECIMENTO DE ENFERMAGEM NO CONTROLE DE INFECÇÃO EM ONCOLOG IAa
}

\author{
Nádia Fontoura SA N H U D O ${ }^{b}$, M arléa Chagas M ORE IRA $^{c}$, Vilma de CA RVA L H O ${ }^{d}$
}

\section{RESUM 0}

O s clientes com câncer apresentam complicações significativas de morbi-mortalidade relacionadas à infecção. E sta revisão integ rativa tem como objetivo analisar a produção científica da enfermagem acerca do controle de infecções nos clientes com câncer. A busca dos 37 artigos incluídos foi por meio eletrônico em bases de dados, no período de 2002 a 2009. Os dados foram anal isados na per spectiva da M etodol ogia de Categorização E pistemológica para a Pesquisa na E nfer magem. O s resultados indicam que a produção é internacional, mais setorizada na área da oncologia clínica, com ênfase nos clientes como sujeitos das investigações, tendência exógena da origem da infecção e prevalência de artigos de revisão de liter atura. Conclui-se que a produção do conhecimento no controle de infecção é prioridade no cenário nacional, as ações devem ser baseadas numa visão sistêmica, considerando a complexidade que envolve os profissionais na prestação do cuidado de enfermagem.

D escritores: Infecção hospitalar. Enfermagem oncológica. Pesquisa em enfermagem.

\section{RESUMEN}

L os clientes con cáncer tienen complicaciones importantes de morbilidad y mortalidad relacionadas con la infección. E sta revisión integradora es analizar la producción científica de los enfermeros sobre el control de infecciones en los clientes con el cáncer. L a búsqueda de los 37 artículos se insertó a través de bases de datos electrónico, en el período 2002 a 2009. L os datos fuer on analizados desdela per spectiva de la metodol ogía epi stemológica para la Investigación en E nfermería. L os resultados indican que la producción es internacional, el área más sectorizado de la oncología clínica, la tendencia fuente exógena de infección y prevalecía de la literatura revisada. Se concluye que la producción de conocimiento en el control de la infección es una prioridad en la escena nacional, las acciones deben basarse en una visión sistémica, teniendo en cuenta la complejidad que implica en el cuidado profesional de enfermería.

D escriptores: Infección hospitalaria. E nfermaría oncológica. Investigación en enfermaría.

T ítulo: Tendencias de la producción de conocimiento de enfermería en el control de la infección en oncología.

\section{ABST RACT}

$\mathrm{H}$ ealth dients with cancer have significant morbimortality complications related to infection. This integrative review analyzes scientific production in nursing about infection control in health clients with cancer. The 37 articles included were found through electronic databases, and published from 2002 to 2009. The data were analyzed from the perspective of epistemological categorization methodology for nursing research. The results indicate that the production is international, that it is mostly in the area of clinical oncology, that it emphasizes customers as subjects of investigation, that it also emphasizes exogenous sources of infection, and that literature review articles are prevalent. Conclusions are that the production of know ledge in infection control is a priority on the national scene, and that actions must be based on a systemic view that considers the complexity of providing professional nursing care

Descriptors: Cross infection. 0 ncologic nursing. N ursing research.

$T$ itle: T rends in knowledge production in nursing about infection control in oncology.

\footnotetext{
a Estudo desenvolvido na D isciplina de Tópicos Especiais em Enfermagem do Programa de Pós-G raduação em Enfermagem da E scola de Enfermagem Anna N ery da Universidade Federal do Rio de Janeiro (EEAN/ U FRJ), Rio de Janeiro, Brasil.

b D outoranda pelo Programa de Pós-G raduação em Enfermagem da EEAN / U F RJ, N úcleo de Pesquisa G estão em Saúde e Exercício Profissional de Enfermagem, Rio de Janeiro, Brasil.

' D outora em Enfermagem, Professora Adjunta da EEAN/UFRJ, Rio de Janeiro, Brasil.

${ }^{\text {b }}$ D ocente Livre em Saúde Pública, Professora E mérita da EEAN / U F RJ, Rio de Janeiro, Brasil.
} 


\section{INT RODUÇÃO}

0 controle de infecção é uma das prioridades da enfermagem em oncologia, visto que o risco de infecção é um dos diagnósticos de enfermagem mais destacados nos clientes com câncer, em decorrência do processo de adoecimento, efeitos de drogas imunossupressoras e procedimentos invasivos terapêuticos ou diagnósticos que geram graus variados de imunossupressão(1); um dos principais fatores de risco para tal diagnóstico, de acordo com a $\mathrm{N}$ orth American $\mathrm{N}$ ursing Diagnosis Association $($ NANDA) (2).

A ocorrência de infecção também está associada na adesão dos profissionais às condutas para prevenção e controle. N essa perspectiva, podese afirmar que a equipe de enfermagem tem destaque na prevenção de risco de infecção e promoção da segurança dos clientes, pela possibilidade de implementar medidas de inter rupção na cadeia de transmissão dos microorganismos de forma endógena e exógena ${ }^{(3)}$.

D ada sua relevância, a infecção é um dos temas prioritários para pesquisa na enfermagem em oncologia na proposta da Oncolog y N ursing Society (ONS) na agenda 2009-2013. N a linha de investigação N ursing-Sensitive Patient Outcomes do referido documento, a infecção é focalizada como evento evitável, destacando-se a necessidade de estudos de avaliação de estratégias para a identificação e prevenção desse evento adverso relacionado ao tratamento(4).

Por certo, a prevenção de infecção é um tema relevante na enfer magem em oncologia, na medida em que clientes com câncer apresentam complicações significativas de morbidades e mortalidade e, as enfermeiras desempenham um importante papel no controle da infecção através da prática, pesquisa e educação(3).

Para tanto, torna-se essencial a investigação das tendências do conhecimento produzido acerca da temática na enfermagem para subsidiar ações mais efetivas. A fim de contribuir, o presente estudo teve como objetivo analisar a produção científica da enfermagem acerca do controle de infecções em clientes com câncer.

\section{MÉTODO}

Trata-se de um estudo bibliográfico com a adoção do método de revisão integ rativa que consis- te na construção de uma análise ampla da literatura, que favorece discussões sobre métodos e resultados de pesquisas, bem como reflexões para 0 desenvolvimento de futuros estudos ${ }^{(5)}$.

Para operacionalizar essa revisão, foram adotadas as seguintes etapas: formulação de uma questão de pesquisa; busca na literatura para identificar o tema escolhido; seleção dos estudos a serem incluídos na revisão; avaliação da literatura; e análise e síntese dos dados ${ }^{(5)}$.

A construção da pergunta de pesquisa é essencial para potencializar e atingir aos objetivos do estudo. As palavras, Paciente, I ntervenção, Comparação e "O utcomes" (PICO), formam o acrônimo PICO, estratégia que auxiliou a construção da questão de pesquisa ${ }^{(6)}$. D efinindo como questão norteadora: Qual a tendência da produção científica da enfermagem acerca do controle de infecções nos clientes com câncer?

Para a busca das produções científicas estabeleceu-se o recorte temporal de 2002 a 2009. A el eição desse período está amparada na demanda da Sociedade em relação ao atendimento à saúde, além do acesso aos serviços, desponta a qualidade oferecida, que tem como pressupostos a valorização das pessoas nos processos. Para o controle de infecção, essa é uma questão emergente desde 0 ano de 2002, quando os órgãos internacionais que traçam as diretrizes da qualidade da assistência à saúde, demonstraram uma preocupação crescente com a adesão dos profissionais às normas preconizadas nas legislações e nas evidências científicas para o controle de infecções( ${ }^{(3)}$.

0 acesso as bases de dados ocorreu pelo portal da Coordenação de Aperfeiçoamento de Pessoal deE nsino Superior (CAPES) na L iteratura Latino-A mericana e do Caribe em Ciências de Saúde (LILACS) e Base de Dados em Enfermagem (BDE N F ), com o cruzamento de Controle de Infecções e Enfermagem Oncológica. No PubM ed e Cumulative Index of $\mathrm{N}$ ursing and Allied $\mathrm{H}$ ealth $\mathrm{L}$ iterature ( $\mathrm{CINAHL}$ ), com cruzamento de I nfection Control and Oncologic $\mathrm{N}$ ursing.

Foram identificadas 66 produções, sendo 46 no PubMed, 20 no CINAHL e nenhuma no LILACS e BDENF. N as quais foi procedida a leitura dos resumos e selecionadas as produções para leitura na íntegra.

E mpregando-se um instrumento de anál ise estruturado, foram destacados os dados de interesse. O ptou-se pelos seguintes critérios de inclusão: pes- 
quisas com acesso na íntegra, publicadas entre 2002 a 2009, nos idiomas inglês, espanhol ou português, que tratam especificamente da temática controle de infecção relacionada à assistência a saúde no cliente com câncer.

Das 66 produções, 29 foram excluídas por apresentar os seguintes critérios: seis não tratam de infecção relacionada à assistência a saúde; dez sem acesso ao artigo na íntegra; sete por não ser pesquisa; um pelo idioma francês; e cinco duplicações nas bases de dados.

Foram incluídos 37 artigos para leitura na íntegra, empregando como referencial teóricometodológico para o agrupamento e análise dos dados, a M etodologia de Categorização E pistemológica para a Pesquisa na E nfermagem. T al metodologia é estruturada a partir de dados de caráter epistemológico, configura-se como um esquema "arquétipo", ou seja, um prototípico que emprega um instrumento de análise estruturado, onde foram destacados os aspectos epistemológicos ${ }^{(7)}$.

Nesse estudo, foram aplicados os seguintes aspectos da categorização: a constituição do tema ou problema da pesquisa e o enquadramento metodológico.

A análise da constituição do tema, nesse estudo, possibilita alcançar as questões epistemológicas relacionadas aos seguintes aspectos: a contribuição setorizada em relação à atuação da enfermagem; a origem das infecções como endógenas e exógenas; identificação dos sujeitos nos estudos (profissionais ou clientes).

Para apresentar a setorização, ou contexto da realidade estudada, foi adotado como ár eas de realização dos estudos: atenção básica, oncologia clínica, oncologia cirúrgica, pediatria, cuidados paliativos e pesquisa clínica, conforme utilizado em estudos de classificação epistemológica da produção do conhecimento da enfermagem oncológica no G rupo de Pesquisa G erência do Processo de Cuidar na Enfermagem em Oncologia ${ }^{(7)}$.

Foi considerada infecção endógena, quando as medidas de controle de infecções são diretamente dirigidas às condições clínicas, que pelo processo de adoecimento e tratamento pode repercutir num quadro infeccioso, bem como ligadas a profilaxia com medicações. A infecção exógena foi ligada aos estudos que favorece estratégias para os profissionais adotarem através de medidas de controle, na transmissão agregada aos fatores do ambiente, como objetos, materiais e veiculação de mi- croorganismos pelos profissionais que prestam 0 atendimento entre os clientes ${ }^{(3)}$.

E $m$ relação à análise da metodologia buscamos apreender as bases utilizadas para enquadramento dos artigos, quanto ao tipo de estudo eabordagem quantitativa ou qualitativa de pesquisa.

\section{RESULTADOS E DISCUSSÃO}

Entre os 37 artigos selecionados, segundo a procedência da produção, nenhuma foi publicada no cenário nacional, com predominância no cenário inter nacional pelos Estados U nidos. Todos os artigos incluídos estavam no idioma inglês. Em relação ao ano de publicação e, respectivamente, 0 seu quantitativo foi encontrado o seguinte resultado: 2002 (2), 2003 (6), 2004 (7), 2005 (3), 2006 (4), 2007 (7), 2008 (4) e 2009 (4).

As revistas que apresentam o maior número de publicações são: Clinical J ournal Oncolog y N ursing com 16 artigos, seguindo com seis artigos 0 J ournal of Pediatric Oncology N ursing e em terceiro com cinco artigos cada, os periódicos Oncology $\mathrm{N}$ ursing Forum e Seminars in Oncology N ursing, e os demais estão distribuídos em outros periódicos em torno de uma a duas publicações. A predominância em períodos especializados demonstra que o controle de infecções é uma temática recorrente nas investigações de enfermeiras oncologistas.

\section{Constituição do tema ou problema da pesquisa}

I dentificar os setores que o conhecimento é produzido, proporciona visibilidade dos cenários que os clientes estão sob maior risco de infecção, e possibilita trabalhar numa abordagem de prevenção com o envolvimento dos clientes, da equipe de enfermagem e demais profissionais da saúde através do desenvolvimento de uma cultura de segurança.

De acordo com a Tabela 1, identifica-se que $67,5 \%$ artigos foram produzidos no setor de oncologia clínica, o qual abrange o tratamento quimioterápico, radioterápico e transplante de células tronco (T CT ). As complicações das terapias intervencionistas estão envoltas pela toxidade da quimioter apia, terapia com corticóide e lesões causadas pela radioterapia(1). A neutropenia é uma consequência da toxicidade da quimioterapia sendo realmente identificado como um fator de risco para doença infecciosa no cliente com câncer ${ }^{(8)}$. 
T abela 1 - Distribuição dos artigos de acordo com a setorização da produção dos estudos. Rio de Janeiro, 2002-2009.

\begin{tabular}{lrr}
\hline Setorização dos estudos & $\mathbf{n}$ & \multicolumn{1}{c}{$\%$} \\
\hline Oncologia Clínica & 25 & 67,5 \\
Oncologia Cirúrgica & - & - \\
Pediatria & 5 & 13,5 \\
Cuidado Paliativo & 1 & 2,7 \\
A tenção Básica & - & - \\
Pesquisa Clínica & - & - \\
Sem Setorização & 6 & 16,2 \\
\hline Total & $\mathbf{3 7}$ & $\mathbf{1 0 0}$ \\
\hline
\end{tabular}

Fonte: $\mathrm{D}$ ados pesquisados nas bases de dados PubM ed e CINA HL.

Os indivíduos submetidos ao T CT apresentam alto risco para desenvolver infecção devido ao quadro de imunossupressão. E sta preocupação é centrada na ameaça de longa duração de infecções oportunistas, que podem persistir meses e anos após o transplante ${ }^{(9)}$.

0 cenário da pediatria aparece com 13,5\% estudos, relacionado ao cateter venoso central $(C V C)^{(10,11)}$, quimioterapia ${ }^{(12)}$, discutem as implicações durante 0 tratamento das crianças.

Os demais $16,2 \%$ artigos abordam outras questões e não apresentam um cenário específico. São artigos que discutem temas relacionado à prática profissional, tais como: identificação das prioridades para pesquisa em enfermagem em oncologia ${ }^{(13)}$; orientações gerais para enfermeiros não especialistas em oncologia cuidar de clientes com câncer ${ }^{(14) ;}$ orientações para prevenir infecções fungicas e melhorar a higienização das mãos ${ }^{(15)}$; discussão sobre a vulnerabilidade do indivíduo com imunossupressão à surtos infecciosos ${ }^{(16)}$; higienização das mãos ${ }^{(17) ;}$ traz as evidências sobre a prevenção de infecção em oncologia(18).

0 cuidado paliativo é discutido em $2,7 \%$ estudos, numa perspectiva da gestão de um cliente com tumor maligno cutâneo, com recomendações de ações para prevenção de infecção através dos cuidados instituídos no manejo do curativo ${ }^{(19)}$.

Quanto à origem da infecção, identificou-se em $56,7 \%$ artigos com tendência à infecção exógena, onde são discutidas questões que envolvem a prática de cuidados de enfermagem, voltadas às ações de controle, a dimensão técnica do trabalho de enfermagem nas experiências dos profissionais ou pesquisas de novas possibilidades de intervenção, conforme consta na T abela 2.
T abela 2 - Distribuição dos artigos de acordo com a origem da infecção. Rio de Janeiro, 2002-2009.

\begin{tabular}{lcc}
\hline Origem da infecção & $\mathbf{n}$ & $\mathbf{\%}$ \\
\hline Endógena & 16 & 43,2 \\
Exógena & 21 & 56,7 \\
\hline Total & $\mathbf{3 7}$ & $\mathbf{1 0 0}$ \\
\hline
\end{tabular}

Fonte: Dados pesquisados nas bases de dados PubM ed e CINAHL.

A prevenção de infecção de origem exógena está relacionada aos clientes submetidos ao T CT (9,20-22), CVC ${ }^{(10,11,23-28)}$, vigilância epidemiológica(16,29-30), higienização das mãos dos profissionais ${ }^{(15,17)}$ e outras questões ${ }^{(13,14,18,31)}$.

É investigada a variação da prática assistencial de enfermagem no T CT para identificar as lacunas entre 0 recomendado e o praticado, onde foi detectado que existe pouca variação das práticas, porém com poucas evidências ${ }^{(21)}$.

E $m$ relação ao CVC, os estudos for necem orientações gerais para sua manipulação, como é possível encontrar as causas de infecções, com o objetivo de instituir medidas corretivas, bem como avaliar a efetividade das intervenções. N esse sentido, um estudo identificou como um programa educativo influenciou na incidência de infecção na corrente sanguínea, trazendo a importância da equipe na instuição de medidas preventivas ${ }^{(10)}$.

As complicações infecciosas decorrentes do CVC foram avaliadas por meio de práticas assistenciais, como o uso de luvas estéril para manipular o cateter, comparado com o uso de luvas não estéril(11), outro estudo avaliou a manutenção por um período maior de permanência da agulha de H uber ${ }^{\circledR}$ no cateter $^{(26)}$. E m ambos os estudos, não foram identificadas alterações na incidência de infecção associada ao cateter.

E m outro estudo foi relatada a criação de um instrumento para a enfermagem ter como referência a respeito dos cuidados e manutenção do CVC, focalizando a implementação das medidas de prevenção de infecção nesse sítio(28).

A heterogeneidade focalizada nos estudos em relação ao CVC se deve a condição indispensável de ações voltadas a prevenção de infecção, na complexidade que envolve a prestação do cuidado de enfermagem, como no caso dos clientes críticos com esse dispositivo, onde a infecção repercute em óbitos e ou aumento significativo do período de inter nação ${ }^{(32)}$. 
$\mathrm{Na}$ abordagem de fontes de infecções, foi adotada nos estudos a vigilância epidemiológica, no sentido de conhecer os microorganismos envolvidos nos processos infecciosos, bem como sua forma de transmissão, como no estudo que determina os níveis de fungos totais e Aspergillus em unidades de transplante de células tronco(29). Outro estudo discute 0 surto com microorganismos potencialmente infectantes em clientes imunodeprimidos ${ }^{(16)}$.

Os estudos apontam para inter venções no controle de infecções como eventos adversos com evidências de higienização das mãos ${ }^{(15,17)}$. Na perspectiva da prática baseada em evidências, procuram-se os resultados de pesquisas para imprimi-los na prática, objetivando dar cientificidade às ações dos profissionais, o que traz implicações para a enfermagem, através da instituição da higienização de mãos, as quais têm demonstrado efetividade, contribuindo significativamente na segurança da assistência prestada ${ }^{(18)}$.

0 percentual de $43,2 \%$ dos artigos assinalou a infecção decorrente de mecanismos endógenos, relacionado a complicação da condição clínica do cliente como determinantes da vulnerabilidade à infecção. São estudos que abordam T CT ${ }^{(33-35)}$, microorganismos $^{(36)}$, quimioterapia ${ }^{(8,37,38)}$, mucosite $\mathrm{e}^{(12,39)}$, leucemia linfocítica crônica ${ }^{(40,41)}$, neutropenia ${ }^{(42-44)}$ e ferida maligna cutânea(19). 0 tratamento medicamentoso é apontado num estudo que fornece orientações para o uso de granulócitos como fator estimulante ${ }^{(45)}$, com a intenção de melhora a imunidade. A pulverização dos temas abordados reflete a complexidade que envolve o controle de infecções nos cuidados de enfer magem no processo de adoecimento dessa clientela.

As neoplasias, todas em si debilitantes, levam ao tratamento quimioterápico, que gera graus variáveis de neutropenia, sendo que a principal complicação no cliente com câncer é a de natureza infecciosa, estando relacionada com a intensidade e o período de imunossupressão, em muitos casos decorrentes da quimioterapia(38).

A severidade da neutropenia induzida pela quimioterapia, apresenta como repercussões 0 comprometimento do cliente, dessa forma os estudos oferecem algumas intervenções para proporcionar mais qualidade de vida nesse período(8).

Os agravos ao sistema imune, repercutem em efeitos colaterais como a mucosite, que rompe barreiras de proteção do organismo, acarreta déficit alimentar e infecções bacterianas secundárias ${ }^{(39)}$.
Além disso, o uso da antibioticoterapia intensifica o desequilíbrio da flora e aumento de leveduras, agrava ainda mais esse quadro. N esta situação a equipe de enfermagem tem importante papel, intensificando os cuidados com higiene bucal e administração correta de antifúngicos como medida de prevenção de infecção ${ }^{(18)}$.

No que tange ao TCT, for nece orientações para subsidiar o manejo de clientes submetidos a essa modalidade terapêutica, a qual desencadeia a neutropenia, e configura-se como um grande fator de risco para doença infecciosa no cliente com câncer ${ }^{(33)}$.

A ocorrência de infecção é uma complicação recorrente nos clientes com câncer, desse modo, 0 enfermeiro deve priorizar estratégias de sensibilização e motivação para conduzir a equipe na perspectiva da prevenção de agravos. Porém constatase que na prática gerencial do enfermeiro, principalmente no âmbito hospitalar, predominam ações voltadas à dimensão técnica, focadas principalmente em atividades de coordenação, supervisão e controle $e^{(46)}$.

N essa concepção, deve-se ter consciência de que os processos educativos para a prevenção e controle da infecção hospitalar precisam ser abordados de forma libertadora e participativa, sem opressão dos profissionais. Através de estratégias que valorizem e promovam participação ativa, dando visibilidade que a adesão às medidas de controle de infecções, está relacionada à ação pessoal e coletiva. Simplesmente repassar protocolos informando as normas a serem seguidas é modelo insuficiente para alavancar as mudanças de atitude profissional ${ }^{(47)}$.

A penas $2,7 \%$ estudos discutem a instituição de programa educativo para os profissionais na adesão aos protocolos que visam a redução da infecção ${ }^{(10)}$. A o considerar que os recursos humanos constituem o principal patrimônio das Instituições prestadoras de assistência à saúde. Destacamos o papel da equipe de enfermagem na implementação das medidas que conduzem à prática de prevenção de infecção em todas as atividades que envolvem os cuidados de enfermagem. Como foi evidenciado no estudo que implementou um abrangente programa educativo, ampliando o conhecimento de enfermeiros acerca dos cuidados para reduzir as taxas de infecções relacionada ao CVC ${ }^{(10)}$, houve redução de $36 \%$ de culturas positivas após a intervenção educativa. 
De acordo com a Tabela 3, a categorização dos estudos que procurou destacar os sujeitos investigados quanto a profissionais ou clientes, encontrou predominância nos clientes como sujeitos das pesquisas. Os clientes em maior foco de investigação, ou seja, os mais vulneráveis em adquirir infecções, são os que estão em processo de T CT e os que utilizam o CVC para a modal idade terapêutica quimioterápica.

T abela 3 - Distribuição dos artigos de acordo com sujeitos envolvidos nos estudos. Rio de Janeiro, 2002-2009.

\begin{tabular}{lrr}
\hline Sujeitos das pesquisas & $\mathbf{n}$ & $\mathbf{\%}$ \\
\hline Clientes & 31 & 83,7 \\
Profissionais & 6 & 16,2 \\
\hline Total & $\mathbf{3 7}$ & $\mathbf{1 0 0}$ \\
\hline
\end{tabular}

Fonte: Dados pesquisados nas bases de dados PubM ed e CINAHL.

Somente $16,2 \%$ artigos focalizam os profissionais, através de estudo que identificou as prioridades para pesquisa em enfermagem que atua na oncologia $a^{(13)}$, outro traz orientações gerais para enfermeiros não especial istas em oncologia atuar com clientes com câncer ${ }^{(14)}$, debatem ações importantes para a pesquisa e geração do conhecimento.

Fato preocupante esse quantitativo, pois a atuação da equipe de enfermagem a partir das meIhores evidências disponíveis para o controle de infecções se estende por questões comportamentais que envolvem os profissionais, o que demanda investigações para aprofundar esse conhecimento.

\section{Enquadramento metodológico}

D entre os artigos analisados em relação ao enquadramento metodológico, conforme consta na T abela 4, verificou-se predominância dos estudos que empregam revisão de literatura; demonstra a necessidade dos enfermeiros sustentarem suas ações em bases conceituais, bem como, a busca por evidências. Os enfermeiros na oncologia tem o resultado otimizado na prevenção de infecção, quando empregam as intervenções baseadas nas meIhores evidências disponíveis ${ }^{(18)}$.

Inicialmente houve a intenção de identificar quais os referenciais teóricos empregados para subsidiar as bases explicativas dos estudos, tendo em vista que a sua apreensão per mite identificar a auto- nomia e fronteiras teórico-metodológicas que configuram o campo epistemológico na enfermagem oncológica(7).

T abela 4 - Distribuição dos artigos de acordo com a metodologia empregada nos estudos. Rio de Janeiro, 2002-2009.

\begin{tabular}{lrr}
\hline Enquadramento metodológico & \multicolumn{1}{c}{ n } & \multicolumn{1}{c}{$\%$} \\
\hline Revisão da literatura & 19 & 51,3 \\
Relato de experiência & 3 & 8,1 \\
Pesquisa quantitativa & 13 & 35,1 \\
Pesquisa qual itativa & 2 & 5,4 \\
\hline T otal & $\mathbf{3 7}$ & $\mathbf{1 0 0}$ \\
\hline
\end{tabular}

Fonte: Dados pesquisados nas bases de dados PubM ed e CINAHL.

De maneira geral, os estudos analisados não apontam os referenciais teóricos adotados, o que pode ser identificado é a significante presença de pesquisas na abordagem quantitativa. Com ênfase na perspectiva do pensamento explicativo, numa tendência ao pensamento positivista, onde 0 pesquisador se ampara em dados estatísticos para analisar a repercussão das estratégias de controle de infecções nas taxas de infecções.

Ressalta-se a carência de estudos com abordagem qualitativa, orientados pelo pensamento compreensivo, que favorece a subjetividade dos profissionais com abrangência da realidade encontrada pelo pesquisador na prática cotidiana para o controle de infecções, incluído a subjetividade dos profissionais.

\section{CONSIDERAÇÕES FINAIS}

A análise da produção do conhecimento da enfermagem oncológica, há evidências de expressiva setorização na oncologia clínica, revela que os clientes considerados de maior risco para adquirir infecções, são os submetidos ao T CT e a quimioterapia. Esta tendência da produção cientifica demonstra que a prioridade na oncologia no controle de infecções, tanto para os clientes adultos quanto pediátricos, é focalizada para os indivíduos neutropênicos.

Ao categorizar os tipos de infecções nos estudos, quanto à origem exógena ou endógena, identificamos que a preocupação dos enfermeiros relativa ao controle de infecções abrange as duas esferas. Porém, identificou-se uma leve tendência na 
prevenção de infecções de origem exógena, através de estratégias de controle, cujas evidências são da higienização das mãos dos profissionais, as boas práticas de manutenção de CVC, e o controle do ambiente por meio da vigilância epidemiológica de microorganismos.

Ainda que em menor demonstração quantitativa, os estudos que abordam as infecções relacionadas aos mecanismos endógenos, apresentam diretrizes para o cuidado de clientes submetidos ao T CT e a quimioterapia, que geram graus variados de neutropenia. A s ações para o controle de infecções enfatizam o reconhecimento prévio dos sinais e sintomas e orientação dos riscos de infecção para esses clientes. Outras medidas também são evidenciadas, como a importância da higiene da cavidade oral na prevenção da mucosite e manejo dos indivíduos com neutropenia em decorrência do processo de adoecimento.

Os estudos apontam uma forte tendência do cliente com câncer como o sujeito de pesquisa. Os estudos que avaliaram o emprego de protocolos pelos profissionais de enfermagem demonstram efetividade na diminuição dos índices de infecções. Questão que se configura como um conhecimento essencial na abordagem do gerenciamento de riscos no controle de infecções. Como também para os enfermeiros na gerência do processo assistencial, pois são estes profissionais que enfrentam o desafio de aplicar junto à equipe as políticas de controle de infecções.

0 conhecimento produzido neste estudo reiterar as tendências internacionais da ONS, que coloca em suas pautas de prioridades, o incentivo às pesquisas acerca do controle de infecções, enfatizando a infecção como um evento evitável. D essa forma, diante da pouca visibilidade da temática no Brasil, torna-se imprescindível aprofundar estudos nessa perspectiva, como forma de contribuir para a pesquisa, a prática e o ensino na enfermagem em oncologia.

\section{REFERÊNCIAS}

$1 \mathrm{~F}$ riese CR. P revention of infection in patients with cancer. Semin Oncol N urs. 2007;23(3):174-83.

$2 \mathrm{~N}$ orth A merican $\mathrm{N}$ ursing Diagnosis A ssociation. Diagnósticos de enfermagem da NA N DA : definições e classificações 2009-2011. Porto Alegre: A rtmed; 2010.

3 A rias $K M$, Soule $B M$. M anual de controle de infecções da A PIC/ J CA H O. Porto A leg re: A rtmed; 2008.
4 Oncology Nursing Society. 2009/ 2013 research agenda [ Internet] . Pittsburgh; 2009 [ cited 2010 Aug 27]; Available from: http:// www.ons.org/ media/ ons/ docs/ research/2009/ 2013onsresearchagendaexecutivesummary.pdf.

5 M endes KDS, Silveira RCCP, Galvão CM . Revisão integrativa: método de pesquisa para a incor por ação de evidências na saúde e na enfer magem. T exto Contexto Enferm. 2008;17(4):758-64.

6 Santos CM C, Pimenta CA M, N obre M RC. A estratégia PICO para a construção da pergunta de pesquisa e busca de evidências. Rev Latino-Am Enfermagem. 2007;15(3):508-11.

7 M oreira M C, Carvalho V, Silva M M, Sanhudo NF, Filgueira M B. Produção de conhecimento na enfermagem em oncologia: contribuição da E scola de Enfermagem A nna N ery. Esc Anna Nery. 2010;14(3):575-84.

8 Padilla G, Ropka M E. Quality of life and chemotherapy-induced neutropenia. Cancer N urs. 2005;28(3): 167-71.

9 Laffan A, Biedrzycki B. Immune reconstitution: the foundation for safe living after an allogeneic hematopoietic stem cell transplantation. Clin J Oncol N urs. 2006;10(6):787-94.

10 H orvath $B$, N orville $R$, Lee D, Hyde A, G regurich $M, H$ ockenberry $M$. R educing central venous catheter-related bloodstream infections in children with cancer. O ncol N urs Forum. 2009;36(2):232-8.

$11 \mathrm{H}$ emsworth S, Selwood K, Saene RV, Pizer B. D oes the number of exogenous infections increase in paediatric oncology patients when sterile surgical gloves are not worn for accessing central venous access devices? E ur J O ncol N urs. 2007;11(5):442-7.

12 Chen CF, Wang RH, Cheng SN, Chang Y C. Assessment of chemotherapy-induced oral complications in children with cancer.J Pediatr Oncol N urs. 2004; 21(1):33-9.

13 Cohen M Z, Harle M, W oll A M , D espa S, M unsell MS. Delphi survey of nursing research priorities. Oncol N urs Forum. 2004;31(5):1011-8.

14 L eon T G, Pase M. Essential oncology facts for the float nurse. M edsurg N urs. 2004;13(3):165-71.

15 Sheridan-Leos N. Oncology care setting design and planning part II: designing healthcare settings to prevent fungal infections and improve handwashing. Clin J Oncol N urs. 2008;12(3):521-5. 
16 Richard J L, Grimes DE . Bioter rorism: Class A agents and their potential presentations in immunocompromised patients. Clin J Oncol N urs. 2008;12(2):295302.

17 Siegel JH, Korniewicz DM. Keeping patients safe: an interventional hand hygienestudy at an oncology center. Clin J Oncol N urs. 2007;11(5):643-6.

18 Zitella LJ, F riese CR, H auser J, G obel BH, W oolery $M, O^{\prime}$ leary $C$, et al. Putting evidence into practice: prevention of infection. Clin J Oncol Nurs. 2006;10 (6):739-50.

19 W oodward L, H aisfield-W olfe M E . M anagement of a patient with a malignant cutaneous tumor.J W ound Ostomy Continence N urs. 2003;30(4):231-6.

20 Dadd G, M cM inn P, M onterosso L. Protective isolation in hemopoietic stem cell transplants: a review of the literature and single institution experience. J Pediatr Oncol N urs. 2003;20(6):293-300.

21 Bevans $M$, T ierney DK, Bruch C, Burgunder M, Castro K, Ford R, et al. Hematopoietic stem cell transplantation nursing: a practice variation study. Oncol Nurs Forum. 2009;36(6):317-25.

$22 \mathrm{~N}$ owlin A, Roman LM. The promise of stem cells. R N . 2005;68(4):48-53.

23 Chaberny IF, Ruseva E, Sohr D, Buchholz S, G anser $A, M$ attner $F$, et al. Surveillance with successful reduction of central line-associated bloodstream infections among neutropenic patients with hematologic or oncologic malignancies. Ann Hematol. 2009;88(9):907-12.

24 A rch P. Port navigation: Iet the journey. Clin J Oncol N urs. 2007;11(4):485-8.

25 Karamanoglu A, Yumuk PF, G umus M , E kenel M , Aliustaoglu M , Selimen D, et al. Port needles: do they need to be removed as frequently in infusional chemotherapy? J I nfus N urs. 2003;26(4):239-42.

26 Penne K. U sing evidence in central catheter care. Semin Oncol N urs. 2002;18(1):66-70.

27 Smith E, W hite S. Hickman line surveillance in oncology. Cancer N urs Practice. 2008;7(2):29-34.

28 H urst SM , Keith BK. Innovative solutions: a collabor ative effort of critical care oncology: the common ground of tubes and lines. Dimens Crit Care N urs. 2005;24(1):37-40.
29 Hensley M E, Ke W, Hayden RT, H andgretinger R, $M$ cCullers JA. L evels of total fungus and A spergillus on a pediatric hematopoietic stem cell transplant unit. J Pediatr Oncol Nurs. 2004;21(2):67-78.

30 E rbay A, Kanyilmaz D, U sE , M umcuoglu I, Karahan ZC, G ozel G, et al. A cluster of K lebsiela pneumonia bacteremia in a radiation oncology of ward. Am J I nfect Control. 2008;36(9):678-80.

$31 \mathrm{M}$ ank $\mathrm{A}$, Van D er Lelie H . Is there still an indication for nursing patients with prolonged neutropenia in protective isolation? A n evidence-based nursing and medical study of 4 years experience for nursing patients with neutropenia without isolation. Eur J Oncol N urs. 2003;7(1):17-23.

$32 \mathrm{~N}$ etto SM , Echer IC, Kuplich N M , Kuchenbecker R, Kessler F. Infecção de cateter vascular central em pacientes adultos de um centro de terapia intensiva. Rev G aúcha E nferm. 2009;30(3):429-36.

33 Rimkus C. A cute complications of stem cell transplant. Semin Oncol N urs. 2009;25(2):129-38.

34 Saria M G, G ossel in-A comb T K. H ematopoietic stem cell transplantation: implications for critical care nurses begin. Clin J Oncol N urs. 2007;11(1):5363.

35 W est F, M itchell SA. E vidence-Based guidelines for the management of neutropenia following outpatient hematopoietic stem cell transplantion. Clin J Oncol N urs. 2004;8(6):601-13.

36 H ooker M. Clostridium Difficile. Clin J Oncol N urs. 2007;11(6):914-24.

37 Reitz LA. D ose-dense chemotherapy for agg ressive non-Hodgkin lymphoma. Cancer N urs. 2006;29(3): 198-206.

$38 \mathrm{M}$ ontoya L. M anaging hematologic toxicities in the oncology patient. J I nfus N urs. 2007;30(3):168-72.

39 Brown CG, W ingard J. Clinical consequences of oral mucositis. Semin Oncol N urs. 2004;20(1):16-21.

40 Lynn A, W illiams M L, Sickler J, Burgess S. T reatment of chronic lymphocytic leukemia with Alemtuzumab: a review for nurses. Oncol Nurs Forum. 2003;30(4):689-94.

41 M oran $M$, Browning $M$, Buckby $E$. N ursing guidelines for managing infections in patients with chro- 
nic lymphocytic leukemia. Clin J Oncol N urs. 2007;11 (6):914-24.

42 Shelton BK. Evidence-based care for the neutropenic patient with leukemia. Semin Oncol N urs. 2003; 19(2):133-41.

$43 \mathrm{M}$ arrs JA. Care of patients with neutropenia. Clin J Oncol Nurs. 2006;10(2):164-6.

$44 \mathrm{~N}$ irenberg $\mathrm{A}, \mathrm{M}$ ulhearn $\mathrm{L}, \mathrm{L}$ in $\mathrm{S}$, Larson $\mathrm{E}$. E mergency department waiting times for patients with cancer with febrile neutropenia: a pilot study. O ncol N urs Forum. 2004;31(4):711-5.
45 Patricia C, Buchsel PC, Forgey A, G rape F B, H amann SS. Granulocyte macrophage colony-stimulating factor: current practice and novel approaches. Clin J Oncol N urs. 2002;6(4):198-205.

46 Santos J LG, G arlet ER, Lima M ADS. Revisão sistemática sobre a dimensão gerencial no trabalho do enfermeiro no âmbito hospitalar. Rev G aúcha Enferm. 2009;30(3):525-32.

47 Fontana RT, L autert L . A prevenção e o controle de infecções: um estudo de caso com enfermeiras. Rev Bras Enferm. 2006;59(3):257-61.

\author{
E ndereço da autora / Dirección del autor / \\ Author's address: \\ Nádia Fontoura Sanhudo \\ Estrada da Graminha, Cond. Parque das Bromélias, \\ Bairro Graminha \\ 36033-120, Juiz de Fora, M G \\ E-mail: nsanhudo@terra.com.br
}

Recebido em: 01/ 10/ 2010

A provado em: 01/ 06/ 2011 